\title{
The rise and fall of Anopheles arabiensis (Diptera: Culicidae) in a Tanzanian village
}

\author{
J.D. Charlwood \\ Ifakara Centre, Tanzania \\ \&
}

Department of Entomology, Wageningen Agricultural University, The Netherlands

\section{J. Kihonda and S. Sama}

Ifakara Centre, Tanzania

\section{P.F. Billingsley}

Department of Biology, Imperial College of Science, Technology and Medicine, London, UK

\section{H. Hadji}

Ifakara Centre, Tanzania

\section{J.P. Verhave}

Department of Medical Microbiology, University of Nijmegen, The Netherlands

\author{
E. Lyimo \\ Ifakara Centre, Tanzania \\ $\&$
}

Department of Entomology, Wageningen Agricultural University, The Netherlands

\section{P.C. Luttikhuizen}

Department of Animal Taxonomy, Wageningen Agricultural University,

The Netherlands

\section{T. Smith}

Department of Public Health \& Epidemiology, Swiss Tropical Institute,

Basel, Switzerland

$$
\text { \& }
$$

Ifakara Centre, Tanzania

Correspondence: Dr. T. Smith, Dept. Public Health \& Epidemiology, Swiss Tropical Institute, PO Box, CH-4002 Basel, Switzerland. 


\begin{abstract}
The continual recruitment of new individuals makes it difficult to study both the survival of multivoltine mosquitoes, and the size of the infectious reservoir in natural populations of malaria vectors. During long-term surveillance of a population of Anopheles gambiae Giles sensu lato in a Tanzanian village by daily light trapping, a temporary dry spell resulted in the cessation of recruitment for a period of 33 days, and a decline in numbers of $A$. arabiensis Patton caught from over 2000 to less than 10 in a sentinel house. Traps placed elsewhere in the village indicated similar proportionate declines although numbers caught varied according to location. A survival rate of $83 \%$ per day was estimated from the rate of population decline. Survival was unrelated to the size of the mosquitoes. The infectious reservoir (the chance of a mosquito acquiring an infection) was estimated to be $2 \%$ per feed. The exploitation of fortuitous events which temporarily eliminate a single stage in the life cycle has general applicability in the study of the bionomics of multivoltine insects.
\end{abstract}

\section{Introduction}

As appreciated by Ross (1911), the intensity of malaria transmission is extremely sensitive to the survival rate of the vector. Various techniques have therefore been used to measure mosquito survival. Perhaps the simplest is to follow a single cohort from emergence to death. This is straightforward in univoltine mosquitoes such as Aedes cantans (Meigen) (Diptera: Culicidae) with very restricted emergence periods (Service, 1977; Packer \& Corbet, 1989; Hoc \& Charlwood, 1990). More elaborate techniques have generally been required in the determination of the age structure of multivoltine tropical disease vectors with overlapping generations (Service, 1993).

If the larvae are eliminated, either experimentally or as a result of natural processes, the dynamics of a multivoltine population may temporarily mimic those of a univoltine one. This happened to a very large population of Anopheles gambiae Giles (sensu lato) (Diptera: Culicidae) in the Kilombero Valley in south-eastern Tanzania at the end of the dry season of 1991. The population was monitored on a daily basis and survival and Plasmodium falciparum (Protozoa) infection rates determined. The nature of the population decline also enabled us to estimate the size of the infectious reservoir.

\section{Materials and methods}

Description of study site

The study took place in the village of Namawala (Ruipa) $\left(8^{\circ} 09^{\prime} \mathrm{S} 36^{\circ} 24^{\prime} \mathrm{E}\right)$ as part of the Kilombero Malaria Project ${ }^{1}$ (Kilombero Malaria Project, 1992; Teuscher, 1993). The village has been described by Charlwood et al. (1995) and Smith et al. (1993). House positions were determined using a satellite global positioning system (Transpack 'Trimble').

\footnotetext{
'The Kilombero Malaria Project is being carried out at the Ifakara Centre (Kilombero District. Tanzania) in collaboration with the Tanzanian National Institute of Medical Research by the Swiss Tropical Institute (Switzerland), Imperial College (England), The Universities of Wageningen and Nijmegen (The Netherlands), and the World Health Organization Immunology Research and Training Centre, Geneva (Switzerland).
}

Three kilometres south of the main population centre there is a hamlet of 15 houses (Kikulukutu) one of which was used as a sentinel house (fig. I).

The area as a whole has two rainy seasons. The main rains start in March and extend through to May and the short rains occur in December and January. A cool, dry season follows the long rains in June and July. As this dry season extends into November temperatures rise, ground water levels drop and there is extensive burning of arable land and bush. Agricultural activity is resumed during the short rains (when rice is planted) and, although surface water remains scarce, because of a raised water table vegetation remains verdant throughout the second hot dry season (February to March) before the long rains.

Rainfall in 1991 was measured at the field laboratory in the village.

\section{Mosquito collections}

Mosquitoes were collected using CDC miniature light traps as described previously (Kilombero Malaria Project, 1991; Smith et al., 1993). In one, the sentinel house in Kikulukutu, a trap was run on an almost nightly basis from March 1990 until April 1993 (when the house fell down). While these collections have provided large amounts of data, this particular paper concerns collections made between February and April 1991. During this time five other light traps were also run in the village on a twice weekly basis.

\section{Mosquito dissections}

The parity status of unselected mosquitoes collected in the sentinel house was determined by examination of the tracheoles of ovaries after being dissected into distilled water and dried, or by examination of ovariolar stalks dissected into saline (Detinova, 1963). An average of 33 mosquitoes were dissected every day.

\section{Mosquito identifications}

The polymerase chain reaction (PCR) method for identification of mosquitoes of the A. gambiae complex (Scott $e t$ al., 1993) was used to identify unselected samples from the light trap on four separate dates. 


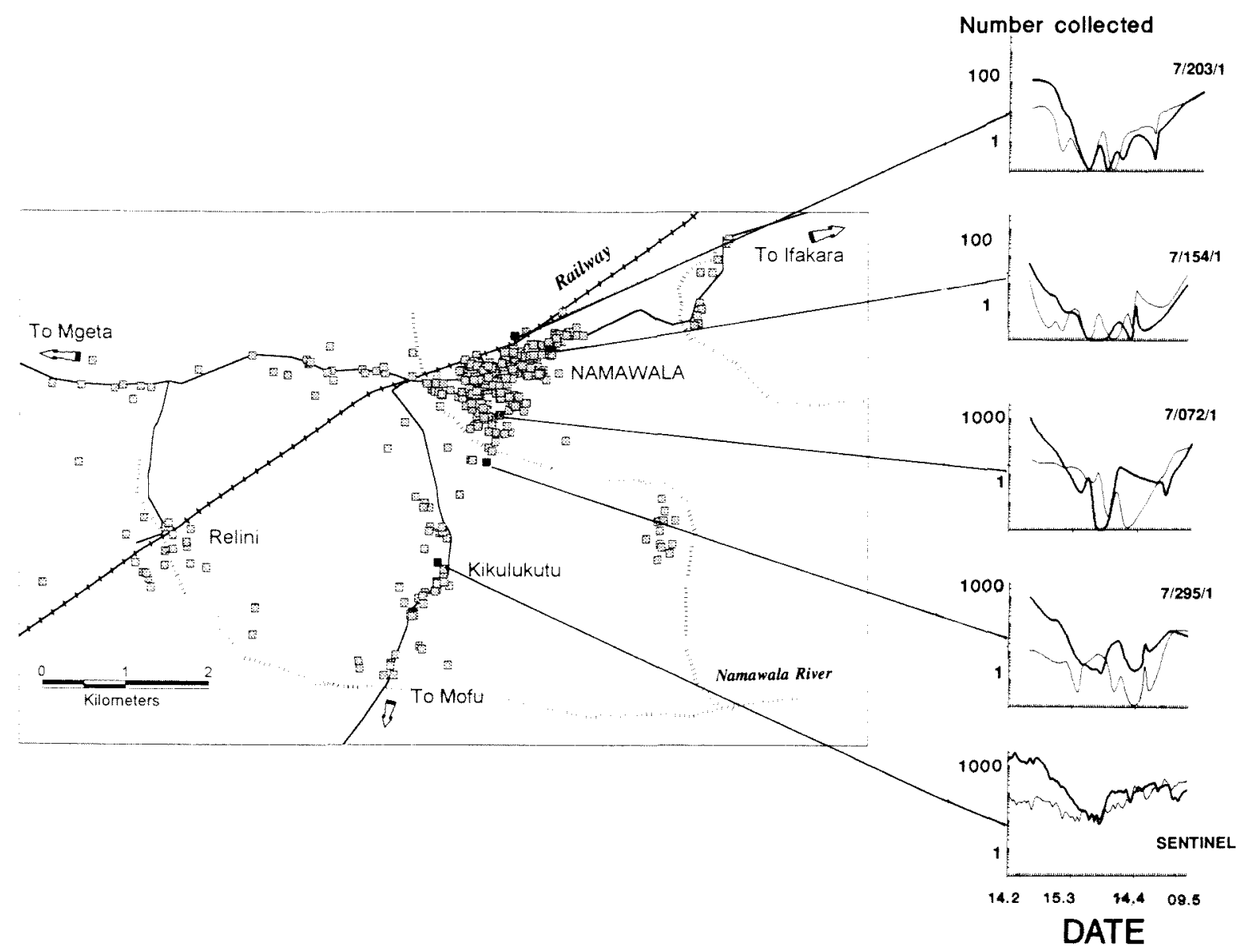

Fig. 1. Map of Namawala village showing positions of all houses recorded in the village 1990-1993, numbers of Anopheles gambiae sensu lato (heavy lines) and A. funestus (thin lines) Giles caught in light traps February-May 1991 and the house positions. The density of houses at any one time is less than that shown in the figure.

\section{Measurement of wing lengths}

Wing lengths, which are a good indicator of mosquito size (Lyimo et al., 1992) were measured for samples of the A. gambiae sensu lato females from the sentinel house. When available at least 25 mosquitoes, were measured each day. This was done to an accuracy of $0.03 \mathrm{~mm}$ using a camera lucida attachment on a stereo-microscope (Lyimo et al., 1992).

\section{Sporozoite ELISA test}

The dried heads and thoraces of the mosquitoes collected from the sentinel house were stored on silica gel in a freezer at $-20^{\circ} \mathrm{C}$ and later examined in ELISA (either in pools of 10 or individually) for the presence of the (NANP) $)_{40}$ repeat region of the Plasmodium falciparum circumsporozoite protein (Burkot et al., 1984). Wells were considered to be positive for sporozoites when the optical density exceeded the mean +3 standard deviations of a set of 6 negative controls on the same plate.

\section{Model for rate of increase in sporozoite prevalence}

The rate of appearance of sporozoites is the same as the rate at which the mosquito population becomes infected assuming that all infections are viable, that survival of the mosquito is independent of infection, and that the mosquitoes are all old enough for the extrinsic cycle to have been completed. Using the estimates in Molineaux (1988), it seems likely that the duration of sporogony in Namawala mosquitoes is less than 2 weeks. We can be confident that most of the mosquitoes from 12 March onwards, i.e. 2 weeks after the start of the decline in numbers, are at least 2 weeks old.

The following difference equations apply to the situation where there is no recruitment of new mosquitoes:

$$
\begin{gathered}
I_{t}=(1-k) I_{t-1}+\Lambda N_{t-1} \\
N_{t}=(1-k-\Lambda) N_{t-1}
\end{gathered}
$$

where: $I_{t}$ is the number of mosquitoes with sporozoites in the cohort on day $t ; N_{t}$ is the number of mosquitoes without sporozoites; $\Lambda$ is the mosquito infection rate (viable infections only) and $\mathrm{k}$ is the mosquito death rate.

Hence the sporozoite rate on day $t\left(\pi_{t}\right)$ is:

$$
\pi_{t}=\frac{I_{t}}{I_{t}+N_{t}}=\frac{(1-k) I_{t-t}+\Lambda N_{t-1}}{(1-k)\left(I_{t-1}+N_{t-1}\right)}
$$


and the sporozoite rate on day $t-I\left(\pi_{\mathrm{t}-1}\right)$ is:

$$
\pi_{t-1}=\frac{I_{t-1}}{I_{t-1}+N_{t-1}}
$$

substituting (4) in (3):

$$
\pi_{\mathrm{t}}=\pi_{\mathrm{t}-1}+\frac{\Lambda\left(1-\pi_{\mathrm{t}-1}\right)}{(1-\mathrm{k})}
$$

so long as most of the mosquitoes remain uninfected, the term $\left(1-\pi_{t-1}\right)$ is close to 1 , and hence we can say:

$$
\pi_{\mathrm{t}} \approx \pi_{\mathrm{r}-1}+\frac{\Lambda}{(1-\mathrm{k})}
$$

We thus expect an approximately linear increase in the sporozoite prevalence during the period of no recruitment, possibly with a lag during the first two weeks. This is equivalent to an additive regression of sporozoite prevalence on time, as opposed to the usual multiplicative logistic regression models commonly fitted to binary data. The additive model was fitted by maximum likelihood within the package EGRET (SERC, 1991).

\section{Results}

During the last ten days of December 1990, at the start of the short rains, a geometric mean of $21.5 \mathrm{~A}$. gambiae sensu lato and $4.2 A$. funestus Giles were caught in the sentinel house in Kikulukutu. Six $A$. gambiae and 13 A. arabiensis were identified from the collection of the 24 January. There was a wave of emergence, lasting for only 1 or 2 days, 7-8 days after rain at the end of January, and in the second week of February when the geometric means reached 2624.5 and 43.1, respectively. A similar peak of emergence occurred 8 days after rain on the 16 and 17 of February. Figure 2 illustrates how dynamic the population was at this time. All 14 mosquitoes identified to species at this time (14 February) were $A$. arabiensis as were all 13 identified from the collection of 2 March, hence we assume that this was the only member of the $A$. gambiae complex collected at the start of this year. Temperatures remained high throughout the study period (with daily maxima above $30^{\circ} \mathrm{C}$ ) and rain on 21 February and 3 March was not sufficient for breeding and was not accompanied by the appearance of pre-gravid or nulliparous insects in the light trap.

The near absence of young $A$. gambiae sensu lato from 28 February to 2 April indicated that there were virtually no new recruits to the population, hence we assume that all were $A$. arabiensis. During the dry period that followed numbers caught in the trap declined in an exponential fashion (corresponding to a straight line when the numbers were log-transformed (fig. 3)). Assuming no net migration, the daily decline in numbers caught between 28 February and 26 March therefore represents the mortality rate of the adults. A log-linear model fitted to the light trap catches from the sentinel house indicated that the decline (on a natural log scale) was 0.191 per day (SE 0.002). This implied that daily survival was $0.827(95 \% \mathrm{Cl})$. Hence, if we assume an exponential survival distribution (which is what the line implies), the life expectancy of $A$. arabiensis during the study period was $1 /(1-0.827)=5.8$ days.

Sporozoite prevalence increased steadily as the mosquito population declined (fig. 3). The fitted line for the additive model is superimposed onto the data (fig. 3). The model estimated a daily increase in sporozoite rate $\left(\pi_{\mathrm{t}}\right)$ of 0.0080 $(\mathrm{SE}=0.0047)$. Using the estimate of the survival rate, $1-k=0.827, \Lambda$ is estimated $0.0080 \times 0.827=0.0066 /$ day. In fact, the line fits the data quite well even when extrapolated back to 4 March, suggesting that even then most of the mosquitoes were old enough for sporogony to have occurred. Following the arrival of the new females into the population from 2 April, the sporozoite rate immediately dropped to less than $3 \%$ from the preceding $20 \%$ peak (data not shown).

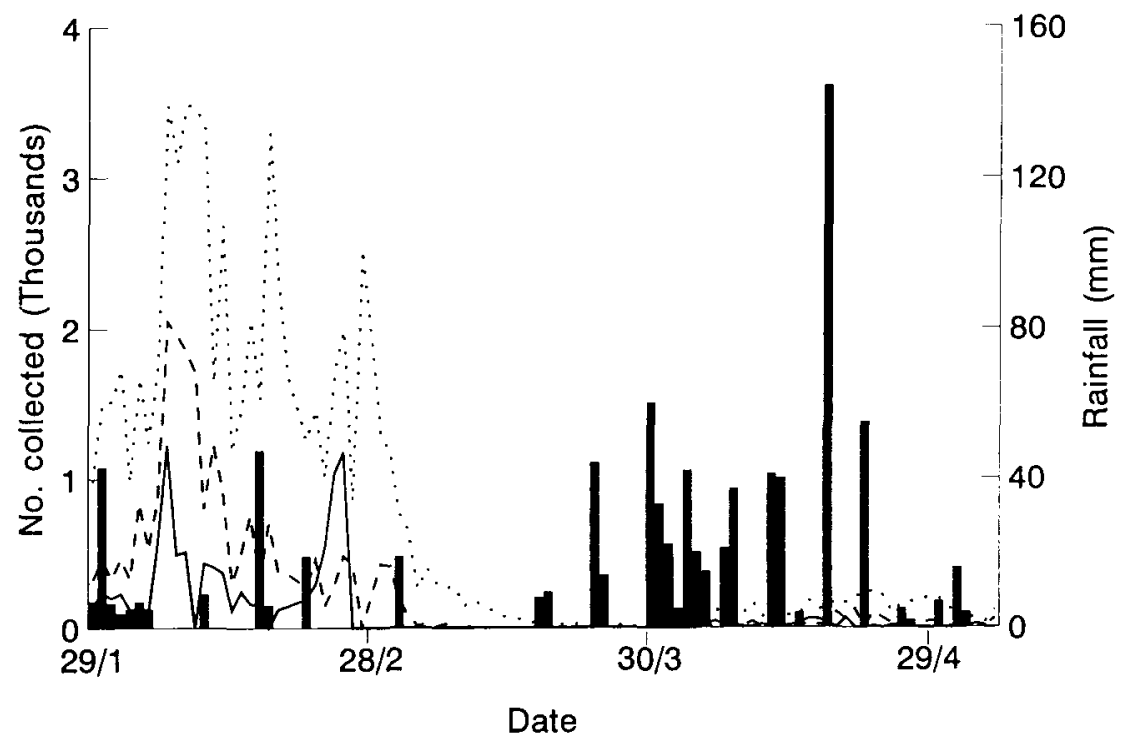

Rainfall - - Nulliparous - Pre-gravid ' Total

Fig. 2. Rainfall and pre-gravid rate of Anopheles arabiensis from the sentinel house, Kikulukutu, January-April 1991. 


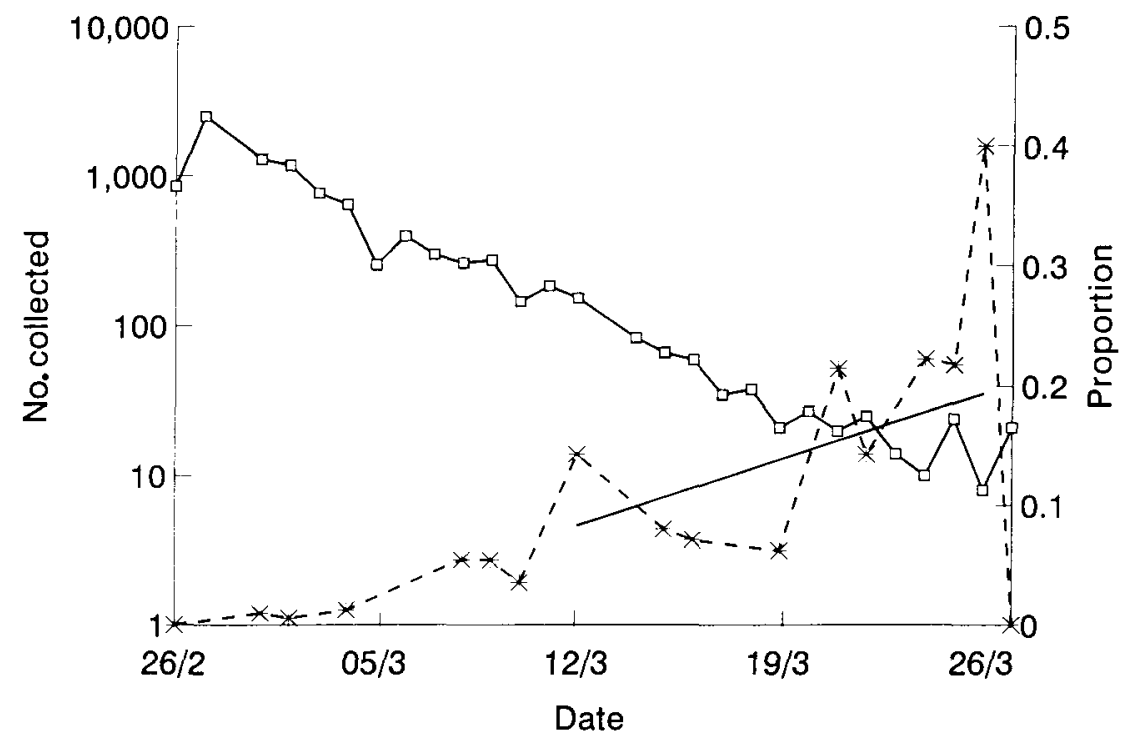

$\rightarrow$ No of $\underline{A \text {. arabiensis }} *$ Sporozoite prevalence - Fitted prevalence

Fig. 3. Population decline and sporozoite rate of Anopheles arabiensis from the sentinel house, February-March 1991.

Average oviposition cycle length (determined by estimation of the proportion of mosquitoes with ovariolar sacs) was estimated to be 2.7 days (Charlwood, unpublished data). Using this figure, the size of the infectious reservoir (the chances of a mosquito taking an infectious meal in any one oviposition cycle) was therefore 2.7 times the daily rate of sporozoite acquisition, i.e. $2.7 \times 0.0066=0.018$
Despite obvious differences in expected average age the mean wing length of the collected $A$. arabiensis remained more or less constant (fig. 4). Size variation was therefore not associated with differential survival.

The appearance of new recruits (pre-gravid females with ovaries at Christophers' (1911) Stage I or N occurred 8 days after the rain on $24 \mathrm{March}$. Although the population of

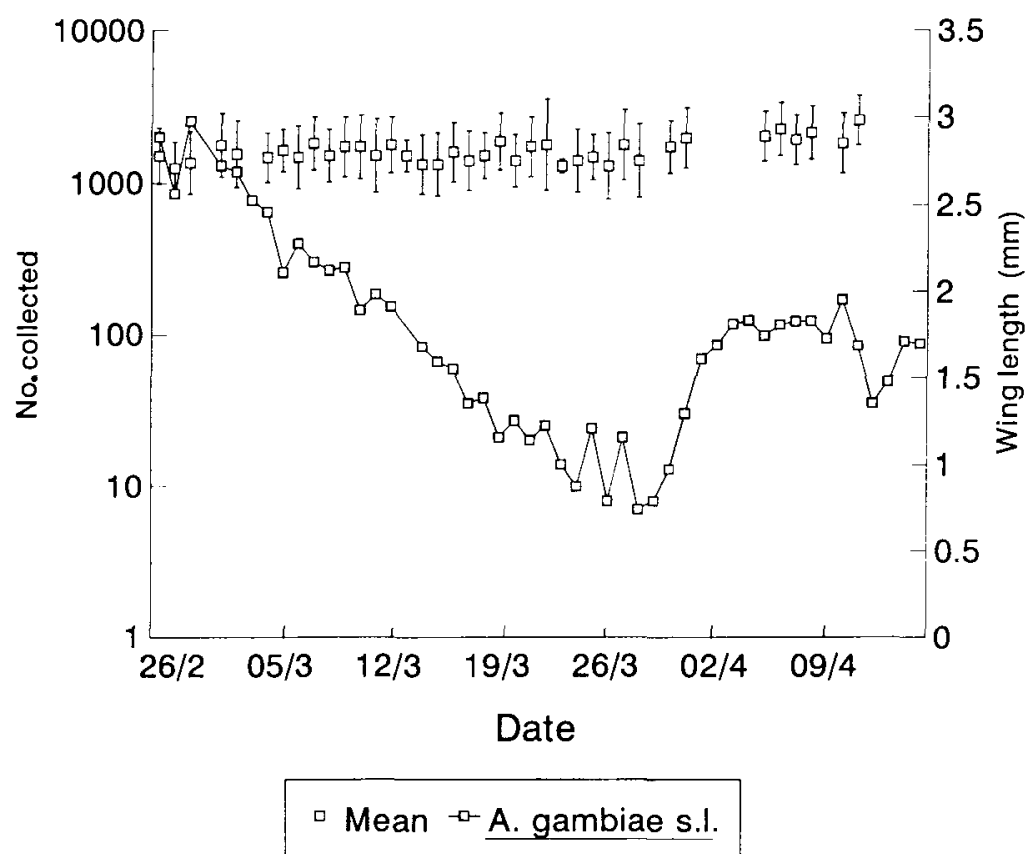

Fig. 4. Wing size of Anopheles arabiensis from the sentinel house February-April 1991. Error bars indicate \pm 1 standard deviation. 
Table 1. Numbers of Anopheles gambiae sensu lato caught in different houses in Namawala from 1-25 March 1991.

\begin{tabular}{lccccc}
\hline House & $\begin{array}{c}\text { No. of } \\
\text { nights } \\
\text { trap } \\
\text { operated }\end{array}$ & $\begin{array}{c}\text { Mean no. } \\
\text { of } \\
\text { o.gambiae } \\
\text { caught }\end{array}$ & $\begin{array}{c}\text { Ratio } \\
\text { to } \\
\text { sentinel } \\
\text { house }\end{array}$ & \multicolumn{2}{c}{$95 \%$ confidence } \\
bounds & for ratio \\
\hline Sentinel & 24 & 262.0 & 1.00 & - & - \\
$7 / 295 / 1$ & 7 & 18.6 & 0.20 & 0.17 & 0.24 \\
$7 / 072 / 1$ & 7 & 56.4 & 0.34 & 0.31 & 0.38 \\
$7 / 154 / 1$ & 7 & 1.6 & 0.009 & 0.005 & 0.017 \\
$7 / 203 / 1$ & 7 & 21.4 & 0.13 & 0.11 & 0.15 \\
\hline
\end{tabular}

${ }^{1}$ Ratio estimated using a log-linear model to allow for the decline in the mosquito population by day.

A. gambiae sensu lato increased at this time to over 100 per night, daily heavy rainfall from 30 March appeared to have a detrimental effect on numbers. The population remained relatively low throughout the remainder of the wet season. Some species replacement obviously occurred since seven A. gambiae were identified from the 19 assayed from 27 April. Further increases in the proportion of $A$. gambiae caught were observed as the wet season progressed (Luttikhuizen, unpublished data). As would be expected the $A$. funestus was less affected by the rain, and numbers, as usual, increased to over 1000 a night at the end of the wet season.

A similar pattern in population variation of A. gambiae sensu lato was observed in other areas of the village (fig. 1) but numbers collected in each trap varied according to location. Those traps near the centre of the village and furthest away from the low lying area of rice cultivation caught fewest mosquitoes (table 1).

\section{Discussion}

Short-term changes in the weather can have dramatic effects on temporary pool breeding mosquitoes as illustrated by $A$. arabiensis in Namawala during 1991. The population changes recorded there represent an archetype of those generally seen during the rains in the Kilombero valley.

The short rains from the end of December to 20 February provided innumerable small surface water pools in the claybased topsoil that acted as transient breeding sites, leading to sharp rises in the pre-gravid rate and a build-up of the adult $A$. arabiensis population to approximately 100 times the dry season average. The drying out of puddles, leading to mass larval mortality, may have been responsible for the dramatic fluctuations in recruitment observed. Unlike the 5 day lag between rain and the appearance of pre-gravid insects recorded by Gillies (1954), the three lags observed in the Namawala A. arabiensis population were each of 8 days duration. This is consistent with a 6 or 7 day immature period and a 1 or 2 day post emergence teneral period. In outlying areas of the village and by the Kilombero River, where most dry season breeding of $A$. arabiensis takes place, many larvae were observed in hippopotamus footprints. However, the water table was not raised and in the dry spell between the two rains such breeding sites evaporated, eliminating the larvae. Environmental conditions for adults, which spend much of their time inside houses remained reasonably constant. This selective elimination of the larvae led to the serendipitous log-linear decline in the adult population which lasted until 2 April. The $19 \mathrm{~mm}$ of rain on 3 March did not interrupt this decline. The duration of the decline was one feature of early 1991 which was unusual. Other declines observed in Namawala have only been sustained in a log-linear fashion for 5-7 days.

Once the main rains started, breeding sites once again became available, and the $A$. gambiae sensu lato population began to build again especially in the periphery of the village where the highest adult densities occurred. However, as the rains progressed, the relationship between rainfall and $A$. gambiae sensu lato emergence became more complex, perhaps because two members of the complex were present and mosquito numbers varied in a more irregular fashion. Unfortunately our understanding of the relationship between the larval ecology of A. gambiae sensu lato and rainfall remains inadequate. Thus the proportion of new recruits (pre-gravid mosquitoes) fell, possibly because many larvae were lost due to flash flooding as the water table rose.

There was no sudden cessation of emergences at the end of the long rains, when cooler temperatures meant that there was relatively little evaporation. The raised water table also meant that conditions changed more slowly than after the short rains and much breeding occurred in shallow ponds.

Both A. gambiae and A. arabiensis are known to occur in the Kilombero valley. It is often thought that the latter species has a lower survival rate than the former (Gillies \& Wilkes, 1965; Gillies \& deMeillon, 1968). Although the population in Namawala may have been mixed, the exponential nature of the decline suggests that at the time of the study both species were behaving similarly. All mosquitoes identified during the decline were $A$. arabiensis. In other areas this species feeds on animals as well as on man. In Namawala virtually all of the mosquitoes from indoor resting collections whose stomach contents have been examined have fed on man (Charlwood et al., 1995) and in the absence of bednets, feeding success is independent of mosquito density. Hence anthropophagic mosquitoes, unlike zoophagic ones, do not suffer from host defensive responses (Charlwood et al., in press). It is possible that animal feeding incurs a higher mortality than feeding on humans.

As would be expected, the $A$. funestus population, which is dependent more on the water table, did not respond as much to the initial period of rainfall. This species becomes more important as a vector towards the end of the long rains when ample shaded breeding sites are available. The biology of this species in Namawala will be discussed elsewhere.

The population decline of $A$. arabiensis in March enabled an estimate of daily survival to be determined; such serendipity eliminated the need for age grading dissections (over 3000 of which would have been needed to produce an equivalent survivorship curve). The close fit to an exponential decay indicated that there was no increase in mortality with age, in contrast to the general pattern found in A. gambiae sensu lato by Clements \& Paterson (1981). Survival rates of members of the $A$. punctulatus Dönitz complex in Papua New Guinea have been found to be Gompertz at one time and exponential at others (Charlwood, 1986). The daily survival rate of $82.7 \%$ obtained in the present paper is almost identical to the $84 \%$ derived by Gillies \& Wilkes (1965) from their classic age grading study in Muheza, on the northern coast of Tanzania, and the figure of $82 \%$ estimated from a more recent study in the same place (Lines et al., 1991). There is a remarkable similarity in survival rates of different anophelines in the tropics (Hii et al., 1990), but the slightly higher survival of those in tropical Africa means 
that they have a considerably higher vectorial capacity (Charlwood et al., 1985).

The average size of the mosquitoes remained the same throughout the population decline and subsequent revival, implying that size differences were not important for survival among adult $A$. arabiensis in agreement with the results of Lyimo (1993) for a nearby village.

By sporozoite and age-grading dissection, Lines et al. (1991) estimated that $21 \%$ of all feeds in Muheza were infectious to mosquitoes, and suggested that this had increased from an earlier estimate of $11 \%$ (Gillies \& Wilkes, 1965) because of natural selection resulting from increased chloroquine use. Despite heavy chloroquine use in Namawala (Teuscher, 1993) the present analysis of increase in sporozoite rates implies that only $1.8 \%$ of the bloodmeals result in infection in A arabiensis. This estimate is also lower than the $4-10 \%$ obtained by delayed oocyst rate dissections from Namawala (Charlwood and Kihonda, unpublished data) The discrepancy between the two estimates for Namawala perhaps implies a low sensitivity in the present ELISA tests. Other studies in East Africa have had conflicting results on the operating characteristics of this assay (Beier et al., 1987; Adungo et al., 1991).

Many of the parameters involved in malaria transmission go through short-term changes in intensity and may vary geographically. Such effects may have led to an unusually low prevalence of viable gametocyte carriers in this study. Subsequent articles will consider the size and dynamics of the infectious reservoir in this population.

\section{Acknowledgements}

We would like to thank the villagers of Namawala for their cooperation, and the National Institute of Medical Research (NIMR) Tanzania for supporting the Kilombero Malaria Project. We would also like to thank the Katrin Agricultural Research Institute for meteorological data. Financial support was provided by the Swiss Directorate for Technical Cooperation and Humanitarian Aid (SDC) and the Directorate General of Development Cooperation (DGIS) of the Dutch government. We would like to thank the Fotolocatie Binnenhaven for help in figure preparation. PFB is supported by the Royal Society, UK, as a University Research Fellow. Research clearance was granted by the Tanzanian Commission for Science and Technology (UTAFITI) as per ref NSR/RCA 90.

\section{References}

Adungo, N.I., Mahadevan, S., Mulaya, N.L., Situbi, A.B. \& Githure, J.I. (1991) Comparative determination of Plasmodium falciparum sporozoite rates in Afrotropical Anopheles from Kenya by dissection and ELISA. Annals of Tropical Medicine and Parasitology 85, 387-394

Beier, J.C., Perkins, P.V., Wirtz, R.A., Whitmire, R.E., Mugambi, M. \& Hockmeyer, W.T. (1987) Field evaluation of an enzyme-linked immunosorbent assay (ELISA) for Plasmodium falciparum sporozoite detection in anopheline mosquitoes from Kenya. American Journal of Tropical Medicine and Hygiene 36, 459-468.

Burkot, T.R., Zavala, F., Gwardz, R.W., Collins, F.H., Nussenzweig, R.S. \& Roberts, D.R. (1984) Identification of malaria-infected mosquitoes by a two site enzyme-linked immunosorbent assay. American Joumal of Tropical Medicine and Hygiene 33, 783-788.

Charlwood, J.D. (1986) Survival rate variation of Anopheles farauti (Diptera: Culicidae) between neighboring villages in coastal Papua New Guinea. Journal of Medical Entomology 23, $361-365$.

Charlwood, J.D., Birley, M.H., Dagoro, H., Paru, R. \& Holmes, P.R. (1985) Assessing survival rates of Anopheles farauti (Diptera: Culicidae) from Papua New Guinea. Joumal of Animal Ecology 54, 1003-1016.

Charlwood, J.D., Smith, T., Kihonda, J., Heinz, B., Billingsley, P. \& Takken, W. (1995) Density independent feeding success of malaria vectors in Tanzania. Bulletin of Entomological Research 85, 29-35.

Clements, A.N. \& Paterson, G.D. (1981) The analysis of mortality and survival rates in wild populations of mosquitoes. Journal of Applied Ecology 18, 373-399.

Christophers, S.R. (1911) The development of the egg follicle in anophelines. Paludism 2, 73.

Detinova, T.S. (1963) Age-grouping methods in Diptera of medical importance, with special reference to some vectors of malaria. World Health Organization Monographs Series 47, $216 \mathrm{pp}$.

Gillies, M.T. (1954) The recognition of age-groups within populations of Anopheles gambiae by the pre-gravid rate and the sporozoite rate. Annals of Tropical Medicine and Parasitology $48,58-74$.

Gillies, M.T. \& de Meillon, B. (1968) Anophelinae of Africa south of the Sahara, (Ethiopian Zoogeographical Region). 2nd edn. Johannesburg, Publication of the South African Institute for Medical Research no. 54.

Gillies, M.T. \& Wilkes, T.J. (1965) A study of the age composition of populations of Anopheles gambiae Giles and $A$. funestus Giles in north-eastem Tanzania. Bulletin of Entomological Research 56, 237-262.

Hii, J.L.K., Birley, M.H. \& Sang, V.Y. (1990) Estimation of survival rate and oviposition interval of Anopheles balabacensis mosquitoes from mark-recapture experiments in Sabah, Malaysia. Medical and Veterinary Entomology 4, 135-140.

Hoc, T.Q. \& Charlwood, J.D. (1990) Age determination of Aedes cantans using the ovarian oil injection technique. Medical and Veterinary Entomology 4, 227-233.

Lyimo, E.O., Takken, W. \& Koella, J.C. (1992) Effect of rearing temperature and larval density on larval survival, age at pupation and adult size of Anopheles gambiae. Entomologia Experimentalis et Applicata 63, 265-271.

Lyimo, E.O. (1993) The bionomics of the malaria mosquito Anopheles gambiae sensu lato in southeast Tanzania. PhD Thesis, Wageningen Agricultural University.

Kilombero Malaria Project (1992) The level of anti-sporozoite antibodies in a highly endemic malaria area and its relationship with exposure to mosquitoes. Transactions of the Royal Society of Tropical Medicine and Hygiene 86, 499504.

Lines, J., Wilkes, T.J. \& Lyimo, E. (1991) Human malaria infectiousness measured by age-specific sporozoite rates in Anopheles gambiae in Tanzania. Parasitology 102, 167-177.

McCullagh, P. \& Nelder, J.A. (1989) Generalised linear models. London, Chapman Hall.

Molineaux, L. (1988) The epidemiology of human malaria as an explanation of its distribution including some implications for its control. pp. 913-998 in Wernsdorfer, W.H. \& McGregor, I. (Eds) Malaria, principles and practice of malariology. Edinburgh, Churchill Livingstone. 
Packer, M.J. \& Corbet, P.S. (1989) Seasonal emergence, hostseeking activity, age composition and reproductive biology of the mosquito Aedes punctor. Ecological Entomology 14, $433-442$.

Ross, R. (1911) The prevention of malaria (2nd Edition). (With addendum on the theory of happenings). London, Murray.

Scott, J.A., Brogdon W.G. \& Collins, F.H. (1993) Identification of single specimens of the Anopheles gambiae complex by the polymerase chain reaction. American Journal of Tropical Medicine and Hygiene 49, 520-529.

SERC (Statistics and Epidemiology Research Corporation) (1991) EGRET: user's manual. Seattle, Statistics and Epidemiology Research Corporation.

Service, M.W. (1977) Ecological and biological studies of Aedes cantans (Meigen) (Diptera: Culicidae) in southern England. Journal of Applied Ecology 14, 159-196.
Service, M. (1993) Mosquito ecology: field sampling methods. 2nd edn. 988 pp. London \& New York, Elsevier.

Smith, T., Charlwood, J.D., Kihonda, J., Mwankusye, S., Billingsley, P., Meuwissen, J., Lyimo, E., Takken, W. Teuscher, T. \& Tanner, M. (1993) Absence of seasonal variation in malaria parasitaemia in an area of intense seasonal transmission. Acta Tropica 54, 55-72.

Teuscher, T. (1993) Household-based malaria control in a highly endemic area of Africa (Tanzania): determinants of transmission and disease and indicators for monitoring-Kilombero malaria project. Memorias do Instituto Oswaldo Cruz 87, Suppl. III, $121-130$

(Accepted 1 August 1994)

(C) CAB INTERNATIONAL, 1995 